\title{
Dinophysis spp. asociadas a detección de toxinas diarreicas (DSTs) en moluscos y a intoxicación diarreica en humanos (Provincia de Buenos Aires, Argentina)
}

Dinophysis spp. associated to detection of diarrheic shellfish toxins (DSTs) in mollusks and to human diarrhoetic disease episode (Buenos Aires Province, Argentina)

\author{
Eugenia A. Sar ${ }^{1,2}$, Inés Sunesen ${ }^{1,2}$, Andrea S. Lavigne ${ }^{1,3}$ y Alejandra B. Goya ${ }^{4}$ \\ ${ }^{1}$ División Ficología, Facultad de Ciencias Naturales y Museo, Universidad Nacional de La Plata, Paseo del Bosque s/n, 1900 \\ La Plata, Argentina. easar@fcnym.unlp.edu.ar \\ ${ }^{2}$ Consejo Nacional de Investigaciones Científicas y Técnicas, CONICET, Argentina \\ ${ }^{3}$ Dirección Provincial de Pesca, Ministerio de Asuntos Agrarios de la Provincia de Buenos Aires, Calle 51 esquina 12, Torre 1, \\ piso 7, (1900), La Plata, Argentina \\ ${ }^{4}$ Departamento de Toxinas Marinas, Laboratorio Regional Mar del Plata, Centro Regional Buenos Aires Sur, SENASA, Aviso \\ Dorrego y Víctimas del 46, (7600), Mar del Plata, Argentina
}

\begin{abstract}
During J anuary and February 2010 lipophilic shellfish toxins were detected in two bivalve species from Villa Gesell and Mar Azul (Argentina) by the mouse bioassay method and potentially toxigenic species of Dinophysis were consistently found with densities of the order of $10^{3}$ to $10^{4} \mathrm{cell}^{-1}$ in the phytoplankton. Additionally, three cases of human gastrointestinal illness associated with the consumption of cooked wedge clams (Donax hanleyanus) collected in Villa Gesell were reported. The consumed shellfish was positive for lipophilic toxins by the mouse bioassay and the human diseases were consistent with diarrhoetic shellfish poisoning (DSP). This is the first record of detection of an outbreak of diarrhoetic shellfish poisoning associated to the presence of Dinophysis species in Argentina. The species identified were Dinophysis acuminata and D. caudata, previously associated to the detection of diarrhoetic toxins in clams (Mesodesma mactroides), wedge clams and mussels (Mytilus edulis) from the coastal waters of Uruguay.
\end{abstract}

Key words: Dinophysis acuminata, Dinophysis caudata, phycotoxins, diarrhoetic shellfish poisoning (DSP)

Resumen. - Durante enero y febrero de 2010 fueron detectadas toxinas lipofílicas en moluscos de Villa Gesell y Mar Azul (Argentina) utilizando el método de bioensayo en ratón y consistentemente fueron halladas especies toxígenas de Dinophysis en densidades del orden de $10^{3}$ a $10^{4} \mathrm{cel} \mathrm{I-1} \mathrm{en} \mathrm{el} \mathrm{fitoplancton.} \mathrm{Adicionalmente,} \mathrm{fueron} \mathrm{reportados} \mathrm{tres}$ casos de pacientes con gastroenteritis afebril asociada a ingesta de berberechos cocidos (Donax hanl eyanus) colectados en Villa Gesell. Los moluscos consumidos resultaron positivos para toxinas lipofílicas por bioensayo en ratón y los síntomas de las intoxicaciones fueron consistentes con los de la «Intoxicación Diarreica por consumo de Moluscos» (DSP). En Argentina, este es el primer reporte de detección de toxinas diarreicas en moluscos asociada a la presencia de especies de Dinophysis. Las especies determinadas fueron Dinophysis acuminata y D. caudata, previamente asociadas a la detección de toxinas diarreicas en almejas (Mesodesma mactroides), berberechos y mejillones (Mytilus edulis) de la costa de Uruguay.

Palabras clave: Dinophysis acuminata, Dinophysis caudata, ficotoxinas, intoxicación diarreica por moluscos (DSP)

\section{INTRODUCCIÓN}

El género de dinoflagelados tecados Dinophysis Ehrenberg presenta varias especies toxígenas. Yasumoto et al. (1980) determinaron que $D$. fortii Pavillard es productora de una toxina a la que denominaron dinofisistoxina, que fue detectada en mitílidos y en fitoplancton, y señalada como productora de un síndrome diarreico al que designaron Intoxicación Diarreica por Moluscos (Diarrhoetic Shellfish Poisoning, DSP ${ }^{1}$ ). Posteriormente, Lee et al. (1989) determinaron que Dinophysis acuminata Claparède et Lachmann, D. acuta Ehrenberg, D. fortii, D. mitra (Schütt)

${ }^{1}$ Los acrónimos utilizados para las toxinas y para el síndrome que ellas producen son anglosaj ones dada la diversidad de acrónimos que se ha generado en español. 
Abe, D. norvegica Claparède et Lachmann, D. rotundata Claparède et Lachmann and D. tripos Gourret son productoras de toxinas diarreicas de moluscos (DSTs). Esta lista de especies toxígenas fue posteriormente incrementada agregándose Dinophysis caudata SavilleKent (Fernández et al. 2001), D. sacculus Stein (Masselin et al. 1992), D. miles Cleve (Marasigan et al. 2001) y D. ovum Schütt (Raho et al. 2008). La confirmación de la toxicidad de las especies de Dinophysis ha sido dificultosa dado que éstas no podían ser exitosamente cultivadas y los análisis debieron ser realizados a partir de células provenientes de arrastres de red de plancton fraccionados, de células aisladas con micropipetas o de floraciones en que la especie fuera dominante. Recientemente, Park et al. (2006) establecieron cultivos de Dinophysis acuminata sorteando así un cuello de botella en el estudio de sus toxinas. A partir del trabajo de Park et al. (2006) es esperable que otras especies del género sean cultivadas y la producción de toxinas de cada una de ellas sea fehacientemente determinada.

Las toxinas lipofílicas de moluscos son un grupo de compuestos poliéteres entre los que se encuentran el ácido okadaico (OA) y sus análogos, las dinofisistoxinas (DTXs), así como las pectenotoxinas (PTXs) y las yesotoxinas (YTXs) (Fernández et al. 2002). De este complejo de toxinas, históricamente consideradas toxinas diarreicas de moluscos, sólo el OA y las DTXs han mostrado un claro efecto diarreogénico debido a su potente acción inhibidora sobre las proteínas-fosfatasas (PP1 y PP2A), lo que a nivel de los enterocitos produce liberación de iones sodio hacia la luz intestinal, con arrastre de agua, y consecuente diarrea (Scoging 1998). En el caso de las PTXs y YTXs no existen datos epidemiológicos que asocien su presencia en los moluscos con intoxicaciones diarreicas en humanos (Aune \& Yndestad 1993, Aune et al. 2002).

El síndrome diarreico por consumo de moluscos (DSP) cursa con trastornos gastrointestinales agudos tales como, náuseas, vómitos, diarrea y dolores abdominales; los síntomas comienzan entre los $30 \mathrm{~min}$ y las $3 \mathrm{~h}$ posteriores al consumo de moluscos contaminados y duran entre 3 y 4 días; la tasa de mortalidad es nula. Los efectos crónicos de las toxinas diarreicas de moluscos en humanos no son bien conocidos, a pesar que se las considera promotoras de tumores en el tracto digestivo sobre la base de experimentos llevados a cabo con ratas y de los resultados de un estudio epidemiológico que vinculó datos de moluscos contaminados con DSTs y cáncer de colon de los habitantes del un área costera de Francia (Aune 2008, Tubaro et al. 2008).
En Argentina no hay reporte previo de acumulación de DSTs en moluscos vinculada a la aparición de Dinophysis en el plancton. Gayoso \& Ciocco (2001) y Gayoso et al. (2002) reportaron toxinas diarreicas en mitílidos en asociación con la presencia del dinoflagelado bentónico Prorocentrum lima (Ehrenberg) Dodge y episodios de intoxicación diarreica por consumo de moluscos en humanos para el Golfo Nuevo, Provincia de Chubut, Argentina.

Los controles sanitarios de toxinas lipofílicas en la Argentina se realizan por el método de bioensayo en ratones (Yasumoto et al. 1984) en concordancia con las normativas dispuestas por la Unión Europea (Decisión 2002/225/CE), aplicadas también en otros países del mundo. Estos ensayos son desarrollados en laboratorios oficiales del Servicio Nacional de Sanidad y Calidad Agroalimentaria (SENASA), organismo responsable del control sanitario de alimentos de origen animal y vegetal que se destinan al consumo interno y a la exportación.

El presente trabajo tiene por objetivo documentar el primer hallazgo de especies de Dinophysis asociadas a toxinas diarreicas en moluscos (DSTs) y a intoxicación diarreica por consumo de moluscos en humanos (DSP), y fue realizado en el marco de un proyecto de monitoreo de algas nocivas toxígenas y de toxinas en moluscos llevado a cabo por miembros de la División Ficología, de la Dirección de Pesca del Ministerio de Asuntos Agrarios de la Provincia de Buenos Aires y del Laboratorio Regional Mar del Plata del SENASA.

\section{Material y MÉtodos}

El material de este estudio fue colectado en las localidades de Villa Gesell y Mar Azul, Provincia de Buenos Aires, Argentina (Fig. 1). Los muestreos de fitoplancton y de moluscos se realizaron quincenalmente entre enero y febrero de 2010 .

Adicionalmente, datos de fitoplancton obtenidos de muestreos realizados desde marzo de 2008 hasta diciembre de 2009 en las localidades de San Clemente del Tuyú, Santa Teresita, Villa Gesell y Mar Azul (Fig. 1), fueron utilizados junto con los de enero y febrero de 2010 para analizar la distribución temporal de las especies de Dinophysis, la densidad alcanzada por éstas y la densidad del fitoplancton total.

Las muestras para el estudio cualitativo de fitoplancton fueron tomadas en la parte superficial de la columna de agua, entre los 0 y $5 \mathrm{~m}$ de profundidad, con red de plancton de $30 \mu \mathrm{m}$ de apertura de malla para concentrar el material, 


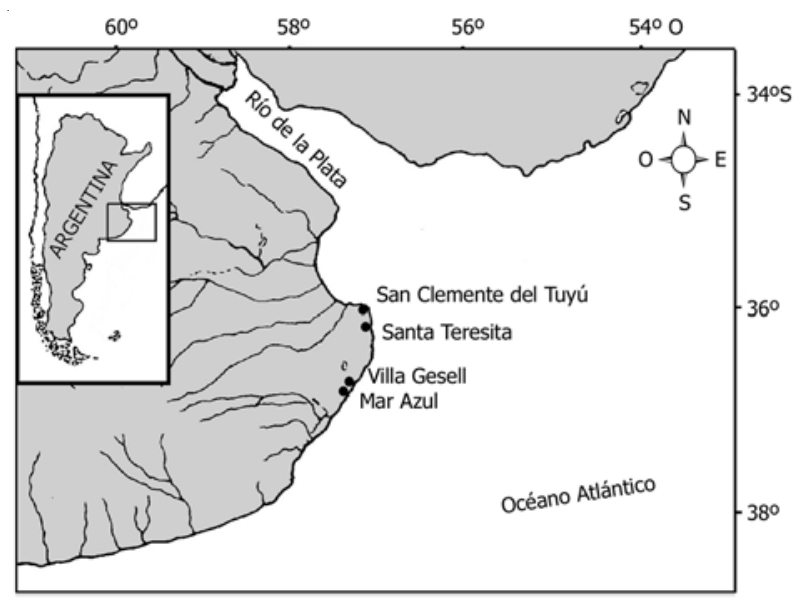

Figura 1. Localización de las estaciones de muestreo en la Provincia de Buenos Aires, Argentina / Location of the sampling stations in Buenos Aires Province, Argentina

y dispuestas en frascos de $125 \mathrm{ml}$ por duplicado. Una de ellas fue inmediatamente fijada con formaldehído al $4 \%$ concentración final y la otra mantenida en frío para su observación in vivo en el laboratorio. Las muestras de agua para el estudio cuantitativo del fitoplancton fueron tomadas con botella Van Dorn, dispuestas en frascos de $250 \mathrm{ml} \mathrm{y}$ fijadas con formaldehído al $0,4 \%$ concentración final.

Las observaciones se realizaron mediante el uso de microscopio óptico con contraste de fases Nikon ${ }^{\circledR}$ Microphot FX, microscopio con contraste interferencial Leica ${ }^{\circledR}$ DM 2500 y microscopio electrónico de barrido Jeol ${ }^{\circledR}$ JSM 6360 LV. Las microfotografías fueron tomadas con los equipos mencionados.

Las muestras cuantitativas fueron analizadas para estimar el número de células por litro con cámara Sedgewick-Rafter ${ }^{\circledR}$ y los recuentos se llevaron a cabo por triplicado. Los datos fueron procesados con el STATISTICAL Software para Windows ${ }^{\circledR}$ 7.0.

El material fue depositado en el Herbario de la División Ficología, Facultad de Ciencias Naturales y Museo, UNLP, bajo los números LPC 11001 al 11180.

La terminología utilizada para la descripción de las especies de Dinophysis y las prescripciones metodológicas para medir los especímenes en vista lateral son las descritas por Balech (2002).

Paralelamente a la recolección de fitoplancton, fueron tomadas muestras de los moluscos Brachydontes rodriguezii d'Orbigny (mejillín) de pilotes del muelle en Villa Gesell y Donax hanleyanus Philippi (berberecho) del intermareal en Mar Azul. Las muestras de moluscos colectadas se mantuvieron congeladas hasta su procesamiento en el Departamento de Toxinas Marinas del Laboratorio Regional Mar del Plata del SENASA. Las especies de moluscos muestreadas fueron las que se encontraban accesibles en el área al momento de realizar la recolección.

Complementariamente, fue analizada una muestra del remanente de los berberechos cocidos consumidos por tres personas que recibieron atención médica en el Hospital de Pinamar por padecer un cuadro agudo, afebril, de vómitos y diarrea, que se inició pocas horas después de la ingestión de los bivalvos. Estos moluscos fueron recolectados en las playas de Villa Gesell a fines de enero y preparados para consumo familiar. La muestra cocida analizada fue obtenida por personal del Departamento de Bromatología de Pinamar a solicitud del Laboratorio del SENASA de Mar del Plata, a fin de investigar un presunto brote tóxico por toxinas diarreicas de moluscos.

Las muestras de moluscos fueron procesadas para la extracción y posterior detección de DSTs mediante bioensayo en ratón (Yasumoto et al. 1984). El método utilizado para la extracción fue el propuesto por Yasumoto et al. (1984) modificado por Yasumoto en Fernández et al. (2002) que permite determinar, en forma conjunta, la presencia o ausencia de toxinas lipofílicas (AO, DTXs, PTXs, YTXs y azaspirácidos AZAs) sobre el cuerpo entero de los moluscos. Todas las muestras de bivalvos fueron previamente analizadas para determinar la presencia de toxinas paralizantes de moluscos (Paralytic Shellfish Toxins, PSTs) por bioensayo en ratón (AOAC 1995).

Los animales de experimentación utilizados fueron ratones albinos, cepa CF1, de $20 \pm 1 \mathrm{~g}$ de peso. El criterio de positividad para toxinas lipofílicas aplicado por el SENASA (Disposición 235/08) es muerte, en menos de 24 $\mathrm{hr}$, de dos de los tres ratones inoculados intraperitonealmente con $1 \mathrm{ml}$ del extracto orgánico equivalente a $25 \mathrm{~g}$ del cuerpo entero de los moluscos (relación peso/volumen: $25 \mathrm{~g}$ de molusco por cada $\mathrm{ml}$ de solución acuosa de Tween 60 al 1\%). Este criterio es concordante con el adoptado por la Unión Europea (Decisión 2002/225/CE).

\section{Resultados}

\section{ANÁLISIS DE FITOPLANCTON}

Las especies de Dinophysis encontradas a partir del análisis de las muestras del área de estudio recolectadas entre marzo de 2008 y febrero de 2010 fueron D. acuminata, 


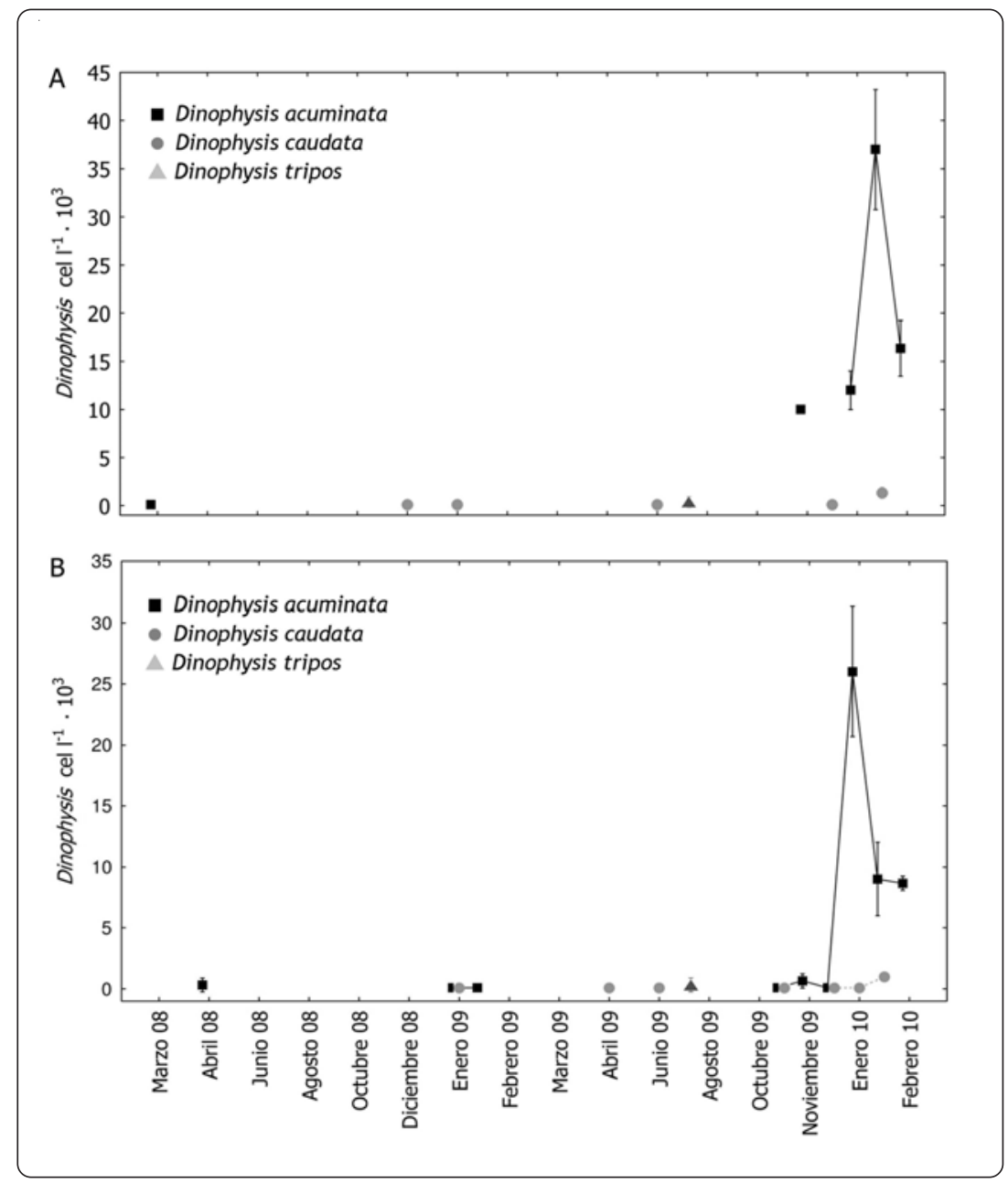

Figura 2. Distribución temporal y densidad de las especies de Dinophysis en A) Villa Gesell y B) Mar Azul. Cada punto representa la media de las tres réplicas y las barras la desviación estándar / Temporal distribution and density of Dinophysis species in A) Villa Gesell and B) Mar Azul. Each point is the mean of three replicas and bars are standard deviation

D. caudata y D. tripos, todas ellas citadas como potenciales productoras de DSTs. Durante este período la frecuencia de aparición de estas especies en las muestras cuantitativas fue mayor en Villa Gesell y Mar Azul que en las restantes estaciones, en las que Dinophysis acuminata apareció en una única

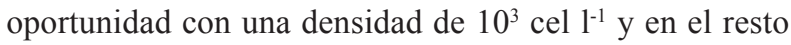
de los casos sólo en las muestras cualitativas. La densidad de las especies de Dinophysis fue variable mensual e interanualmente tanto en Villa Gesell como en Mar Azul (Fig. 2 A y B). Dinophysis acuminata estuvo presente esporádicamente en el año 2008, apareció más frecuentemente en 2009 y alcanzó densidades del orden

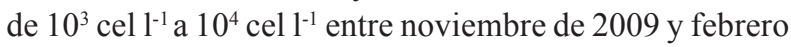
de 2010. En el caso de Dinophysis caudata su aparición en las muestras cualitativas fue más esporádica en Villa Gesell que en Mar Azul y los únicos registros en las muestras cuantitativas fueron de finales de enero de 2010 con una densidad de $10^{3}$ cel $1^{-1}$. Dinophysis tripos fue detectada en julio de 2009 en todas las estaciones de muestreo, alcanzando una densidad de $3 \cdot 10^{2}$ cel $1^{-1}$ en Villa Gesell y Mar Azul, mientras que en San Clemente y Santa Teresita se las detectó sólo en las muestras cualitativas.

La densidad de fitoplancton total también fue altamente variable mensual e interanualmente en Villa Gesell y en Mar Azul (Fig. 3 A y B). Durante enero y febrero de 2010, período en que Dinophysis acuminata alcanzó las mayores densidades, el fitoplancton de Villa Gesell y Mar Azul estuvo dominado por diatomeas y en algunos casos codominado por fitoflagelados nanoplanctónicos no determinados (Tabla 1). Las diatomeas que alcanzaron las mayores densidades fueron Asterionellopsis glacialis (Castracane) Round y un grupo de especies nanoplanctónicas correspondientes a la Clase Coscinodiscophyceae Round \& Crawford (diatomeas céntricas), Rhizosolenia imbricata Brightwell, Leptocylindrus minimus Gran, Leptocylindrus danicus Cleve, Skeletonema sp. y Thalassionema sp., con variantes en la densidad relativa entre estaciones y fechas de muestreo (Tabla 1). En esta tabla sólo fueron 
Figura 3. Distribución temporal y densidad del fitoplancton total en A) Villa Gesell y B) Mar Azul. Cada punto representa la media de las tres réplicas y las barras la desviación estándar / Temporal distribution and total phytoplankton density in A) Villa Gesell and B) Mar Azul. Each point is mean of three replicas and bars are standard deviation

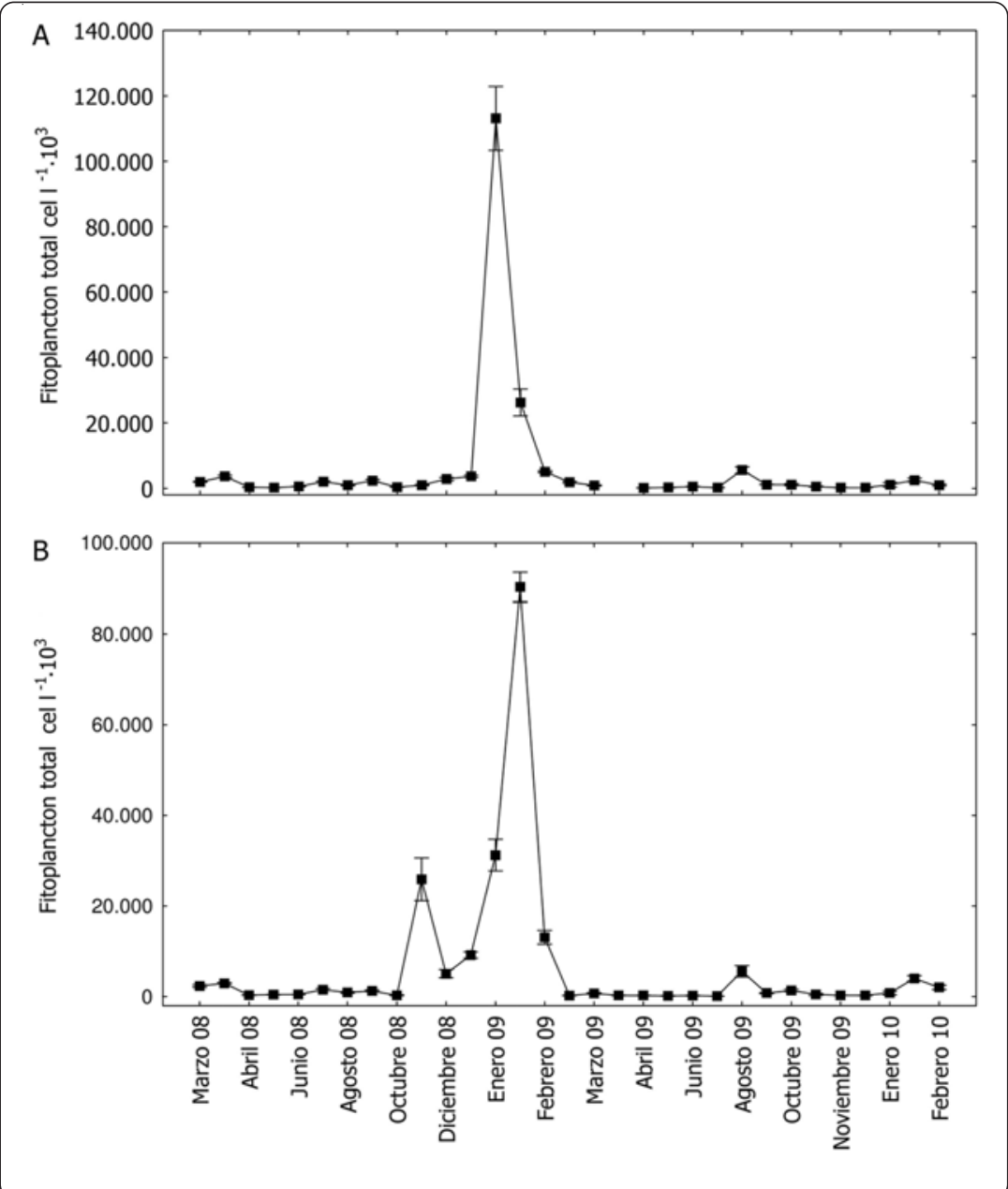

presentadas las especies que alcanzaron densidades del

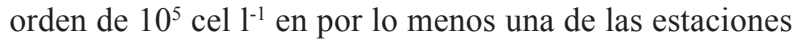
o uno de los muestreos, excepto en el caso de las especies potencialmente toxígenas que pueden acumular ficotoxinas en moluscos a muy bajas densidades, tales como Dinophysis spp. y Gymnodinium catenatum Graham. La contribución relativa de Dinophysis acuminata a la abundancia total de fitoplancton para estos meses osciló entre el 0,22 y el $2,41 \%$ y la de $D$. caudata entre el 0,02 y el $0,06 \%$ (Tabla 1 ).

\section{Caracterización de las especies de Dinophysis ASOCIADAS A LA DETECCIÓN DE DSP EN MOLUSCOS}

\section{Dinophysis acuminata Claparède et Lachmann}

Las características morfológicas de los ejemplares observados (Fig. 4 A-E) coinciden con las de los analizados por Faust \& Gulledge (2002) y Balech (2002). Los especímenes hallados muestran la aleta sulcal izquierda bien desarrollada, extendida más allá del punto medio de la célula, con frecuencia plegada hacia la derecha por detrás de $R_{2} y$ con $R_{2}$ más próxima a $R_{1}$ que $a R_{3}$. La superficie de las placas tecales está ornamentada por areolas circulares con un poro, poros en depresiones someras, y poros no ubicados en depresiones hacia el extremo antapical. Las dimensiones de las células fueron ligeramente inferiores a las descriptas en Faust \& Gulledge (2002) y estuvieron dentro de los siguientes rangos: L (largo), 31,5-38 $\mu \mathrm{m} ; \mathrm{H}$ (ancho dorso-ventral de la hipoteca), 21,3-28 $\mu \mathrm{m}$; E (ancho dorso-ventral de la epiteca), 7-12 $\mu$ m; relación $\mathrm{L} / \mathrm{H}, 1,28$ 1,78, más coincidentes con los datos presentados por Hallegraeff \& Lucas (1988).

\section{Dinophysis caudata Saville-Kent}

Las características morfológicas y dimensiones de los ejemplares observados (Fig. 5 A-C) coinciden con las de 

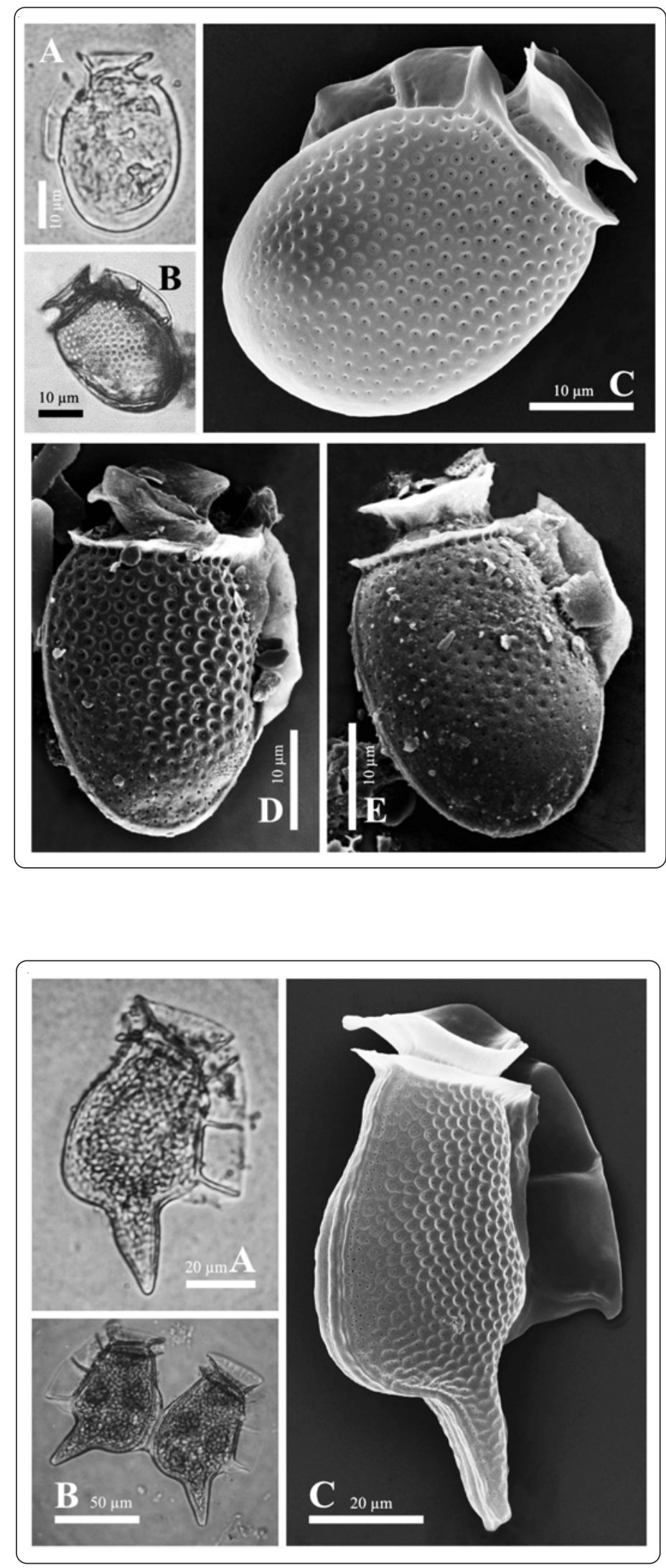

Figura 4. Dinophysis acuminata. A-B. Microscopio óptico (MO). C-E. Microscopio electrónico de barrido (MEB). A-E. Especímenes en vista lateral. C. Vista lateral izquierda. D, E. Vista lateral derecha. Note la pequeña aleta sulcal derecha / Dinophysis acuminata. A-B. Light microscopy (LM). C-E. Scanning electron microscopy (SEM). A-E. Specimens in lateral view. C. Left lateral view. D, E. Right lateral view. Note the small right sulcal list

Figura 5. Dinophysis caudata. A-B. Microscopio óptico (MO). C. Microscopio electrónico de barrido (MEB). A-C. Especímenes en vista lateral. B. Duplete de células provenientes de la fisión binaria. C. Vista lateral derecha. Note la pequeña aleta sulcal derecha / Dinophysis caudata. A-B. Light microscopy (LM). C. Scanning electron microscopy (SEM). A-C. Specimens in lateral view. B. Paired cells from a binary fission. C. Right lateral view. Note the small right sulcal list 
Tabla 1. Densidad del fitoplancton total, densidad relativa de las especies potencialmente

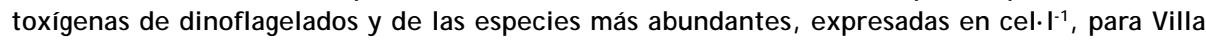
Gesell y Mar Azul en los muestreos de enero y febrero / Total phytoplankton density and relative density of the potentially toxigenic dinoflagellates species and more abundant species, expressed in cell $\left.\cdot\right|^{-1}$, from Villa Gesell and Mar Azul samplings in J anuary and February

\begin{tabular}{|c|c|c|c|c|c|c|}
\hline & \multicolumn{2}{|c|}{ Muestreo 04-01-10 } & \multicolumn{2}{|c|}{ Muestreo 28-01-10 } & \multicolumn{2}{|c|}{ Muestreo 12-02-10 } \\
\hline & Villa Gesell & Mar Azul & Villa Gesell & Mar Azul & Villa Gesell & Mar Azul \\
\hline \multicolumn{7}{|l|}{ Dinoflagelados } \\
\hline Dinophysis acuminata & $1,20 \cdot 10^{4}$ & $2,60 \cdot 10^{4}$ & $4,00 \cdot 10^{4}$ & $9,00 \cdot 10^{3}$ & $1,63 \cdot 10^{4}$ & $8,67 \cdot 10^{3}$ \\
\hline Dinophysis caudata & & & $1,67 \cdot 10^{3}$ & $1,00 \cdot 10^{3}$ & & \\
\hline Gymnodinium catenatum (quistes) & & & & $8,33 \cdot 10^{3}$ & & \\
\hline \multicolumn{7}{|l|}{ Diatomeas } \\
\hline Asterionellopsis glacialis & $1,67 \cdot 10^{4}$ & $2,83 \cdot 10^{5}$ & $6,75 \cdot 10^{5}$ & $2,11 \cdot 10^{6}$ & $2,50 \cdot 10^{5}$ & $1,32 \cdot 10^{6}$ \\
\hline Leptocylindrus danicus & & & $1,83 \cdot 10^{5}$ & $6,67 \cdot 10^{4}$ & $8,33 \cdot 10^{4}$ & $1,33 \cdot 10^{5}$ \\
\hline Leptocylindrus minimus & & & & & $2,25 \cdot 10^{5}$ & $1,90 \cdot 10^{5}$ \\
\hline Rhizosolenia imbricata & & & $8,08 \cdot 10^{5}$ & $7,42 \cdot 10^{5}$ & & \\
\hline Skeletonema sp. & $1,00 \cdot 10^{5}$ & $5,00 \cdot 10^{4}$ & $3,33 \cdot 10^{4}$ & & & \\
\hline Thalassionema sp. & $1,00 \cdot 10^{5}$ & $3,33 \cdot 10^{4}$ & & $5,00 \cdot 10^{4}$ & & \\
\hline Diatomeas céntricas no determinadas & $9,92 \cdot 10^{5}$ & $5,01 \cdot 10^{5}$ & $3,25 \cdot 10^{5}$ & $6,00 \cdot 10^{5}$ & $2,40 \cdot 10^{5}$ & $2,10 \cdot 10^{5}$ \\
\hline \multicolumn{7}{|l|}{ Fitoflagelados } \\
\hline Fitoflagelados no determinados & $1,83 \cdot 10^{5}$ & $1,17 \cdot 10^{5}$ & $2,50 \cdot 10^{4}$ & $1,00 \cdot 10^{5}$ & $1,67 \cdot 10^{4}$ & $1,67 \cdot 10^{4}$ \\
\hline Fitoplancton total & $1,44 \cdot 10^{6}$ & $1,08 \cdot 10^{6}$ & $2,51 \cdot 10^{6}$ & $4,14 \cdot 10^{6}$ & $1,00 \cdot 10^{6}$ & $2,00 \cdot 10^{6}$ \\
\hline Contribución relativa de $D$. acuminata & $0,83 \%$ & $2,41 \%$ & $1,60 \%$ & $0,22 \%$ & $1,63 \%$ & $0,43 \%$ \\
\hline Contribución relativa de $D$. caudata & & & $0,06 \%$ & $0,02 \%$ & & \\
\hline
\end{tabular}

los analizados por Faust \& Gulledge (2002) y Balech (2002). Los especímenes hallados muestran la superficie de las placas tecales areoladas, con un poro por areola. Las dimensiones de las células estuvieron dentro de los siguientes rangos: L (largo), 76-87 $\mu \mathrm{m} ; \mathrm{H}$ (ancho dorsoventral de la hipoteca), 35-43,3 $\mu \mathrm{m}$; $\mathrm{E}$ (ancho dorso-ventral de la epiteca), 7-12 $\mu \mathrm{m}$; relación $\mathrm{L} / \mathrm{H}, 1,28-1,78$, algo más estrechos que los de la literatura citada.

\section{DeteCCIÓN DE TOXINAS EN MOLUSCOS POR BIOENSAYO EN RATÓN}

\section{BioENSAYOS PARA DSP}

Los extractos obtenidos a partir del cuerpo entero de los berberechos provenientes de la muestra fresca de enero y los correspondientes a la muestra cocida sospechosa de provocar los casos de intoxicación en humanos, produjeron la muerte del $100 \%$ de los ratones inoculados. El tiempo de supervivencia estuvo entre 60 y 75 min post-inoculación (Tabla 2). El extracto de berberecho proveniente de muestra fresca de febrero, produjo la muerte del $66 \%$ de los ratones inoculados y el tiempo de supervivencia estuvo comprendido entre 115 a 130 min post-inoculación (Tabla 2). El extracto obtenido a partir del cuerpo entero de mejillines produjo la muerte de todos los ratones inoculados y el tiempo de supervivencia fue de 115 a 235 min post-inoculación (Tabla 2).
Los signos observados en los ratones inyectados intraperitonealmente fueron similares en todos los casos y consistieron en: debilidad generalizada, postración, hipotermia y un leve movimiento convulsivo al que sobrevino la muerte. Estos signos son concordantes con lo descrito por Fernández et al. (2002, tabla 3) para toxinas diarreicas.

\section{Bioensayos PARA PSP}

Las toxinas paralizantes de moluscos (PSTs) no fueron detectables en ninguna de las muestras de moluscos analizadas para el período en que se detectaron toxinas diarreicas.

\section{Discusión}

Dinophysis acuminata y D. caudata alcanzaron las densidades más altas de los últimos dos años durante los meses de enero y febrero de 2010. Éstas tuvieron un orden de magnitud por encima de las concentraciones mínimas a las que según Reguera (2002) las especies de Dinophysis serían capaces de provocar niveles de toxinas en moluscos que los tornan no aptos para el consumo humano. Concordantemente, en esa época fue detectada la presencia de toxinas lipofílicas en berberecho. Por el contrario, en el mes de febrero sólo se observó en el fitoplancton a Dinophysis acuminata y como en el caso anterior, fue detectada la presencia de toxinas lipofílicas en berberecho 
Tabla 2. Resumen de los resultados obtenidos por bioensayo en ratones para detectar DSP / Summary of the results obtained by DSP mouse bioassay

\begin{tabular}{|c|c|c|c|c|c|c|}
\hline Moluscos analizados & Origen & 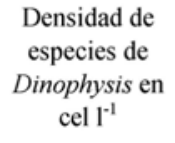 & $\begin{array}{l}\text { Resultado } \\
\text { bioensayo }\end{array}$ & $\begin{array}{c}\text { Relación ratones } \\
\text { muertos /ratones } \\
\text { inoculados }\end{array}$ & Signos en ratones & $\begin{array}{l}\text { Tiempo de } \\
\text { supervivencia } \\
\text { min }\end{array}$ \\
\hline $\begin{array}{l}\text { Berberechos } \\
28 / 01 / 10\end{array}$ & Mar Azul & $\begin{array}{c}\text { D. acuminata } \\
9 \cdot 10^{3} \\
\text { D. caudata } \\
1 \cdot 10^{3}\end{array}$ & Positivo & $3: 3(100 \%)$ & $\begin{array}{l}\text { Debilidad general, } \\
\text { postración, hipotermia, } \\
\text { convulsión premortem, } \\
\text { muerte }\end{array}$ & $60-75$ \\
\hline $\begin{array}{l}\text { Berberechos } \\
12 / 02 / 10\end{array}$ & Mar Azul & $\begin{array}{l}\text { D. acuminata } \\
8,7 \cdot 10^{3}\end{array}$ & Positivo & $2: 3(66 \%)$ & $\begin{array}{l}\text { Debilidad general, } \\
\text { hipotermia, postración, } \\
\text { diarrea, convulsión } \\
\text { premortem, muerte }\end{array}$ & $115-130$ \\
\hline $\begin{array}{l}\text { Berberechos cocidos } \\
25 / 01 / 10 \text { al } 28 / 01 / 10\end{array}$ & Villa Gesell & $\begin{array}{c}\text { D. acuminata } \\
9 \cdot 10^{3} \\
\text { D. caudata } \\
1 \cdot 10^{3}\end{array}$ & Positivo & $3: 3(100 \%)$ & $\begin{array}{l}\text { Debilidad general, } \\
\text { hipotermia, postración, } \\
\text { convulsión premortem, } \\
\text { muerte }\end{array}$ & $60-65$ \\
\hline $\begin{array}{l}\text { Mejillín } \\
12 / 02 / 10\end{array}$ & Villa Gesell & $\begin{array}{c}\text { D. acuminata } \\
1,63 \cdot 10^{4}\end{array}$ & Positivo & $3: 3(100 \%)$ & $\begin{array}{l}\text { Debilidad general, } \\
\text { hipotermia, postración, } \\
\text { convulsión premortem, } \\
\text { muerte }\end{array}$ & $115-235$ \\
\hline
\end{tabular}

y mejillín. Dinophysis acuminata puede ser entonces claramente asociada al episodio por estar presente en ambos muestreos durante el brote de toxicidad en moluscos, aunque es posible que $D$. caudata haya contribuido en una proporción menor a la producción de toxinas durante el mes de enero. Dinophysis acuminata y D. caudata fueron previamente asociadas a la detección de toxinas diarreicas en almeja (Mesodesma mactroides), berberecho (Donax hanleyanus) y mejillón (Mytilus edulis) durante los períodos estivales de 1992, 1994 y 1996 para las aguas costeras de Uruguay (Méndez \& Ferrari 2002).

El cuadro clínico de los intoxicados luego de la ingestión de berberechos provenientes de Villa Gesell resultó consistente con el síndrome DSP, el análisis mediante bioensayo de los moluscos ingeridos produjo resultado positivo para toxinas lipofílicas y las especies de Dinophysis productoras de DSTs fueron detectadas en el fitoplancton al momento de la recolección de los moluscos consumidos. Se descartó la posibilidad de que la etiología de los desórdenes digestivos de los pacientes fuera bacteriana considerando adicionalmente que la gastroenteritis era afebril y que los moluscos consumidos estaban cocidos.

La presencia de PTXs, YTXs, y AZAs a niveles superiores a los regulados por la legislación europea puede ser descartada en función de los signos presentados por los ratones inoculados. Las toxinas mencionadas producen signos similares a los que provocan las toxinas paralizantes (PSTs) (vide en Fernández et al. 2002, tabla 3). Adicionalmente, los azaspirácidos, que son los únicos de este grupo de toxinas lipofílicas que han sido asociados a gastroenteritis aguda en humanos por Satake et al. (1998), son producidos según Krock et al. (2009) por una especie de dinoflagelado tecado recientemente descrita por Tillman et al. (2009) bajo el nombre de Azadinium spinosum Elbrächter \& Tillmann, que estuvo ausente en las muestras del área de estudio al momento de ocurrir el brote de toxicidad en moluscos y en los meses previos.

Sobre la base de lo antes expuesto es posible asociar este episodio de toxicidad en moluscos con la presencia de concentraciones críticas de Dinophysis acuminata y $D$. caudata para el mes de enero y de D. acuminata para el mes de febrero; establecer que la intoxicación en humanos fue provocada por toxinas lipofílicas procedentes de los moluscos ingeridos y presumir que las toxinas implicadas fueron $\mathrm{AO}$ y/o análogos (DXTs) por los signos que presentaron los ratones durante los bioensayos y los síntomas que padecieron las personas intoxicadas.

\section{Agradecimientos}

Los autores deseamos agradecer al Dr. A. Franetovich, Ministro de Asuntos Agrarios de la Provincia de Buenos 
Aires, al Sr. J.D. Novero, Director Provincial de Pesca de la Provincia de Buenos Aires y al Dr. G. Meléndez, Coordinador General Regional del Centro Regional Buenos Aires Sur del SENASA por el apoyo logístico y técnico brindado al proyecto de monitoreo en ejecución. Asimismo, queremos agradecer a Noelia D'Amico e Ignacio Inchaurrondo, estudiantes avanzados de Ingeniería Química, por su cooperación en la preparación de las muestras para análisis de toxinas durante su pasantía en el Laboratorio del SENASA, y al Sr. Christian Maurs, pescador artesanal, por su colaboración desinteresada en la obtención de muestras de moluscos. Finalmente expresamos nuestro agradecimiento a los evaluadores anónimos que con su generoso aporte de ideas y observaciones críticas nos permitieron corregir y mejorar el manuscrito. Este trabajo fue realizado con financiamiento del Consejo Federal Pesquero, Acta CFP $\mathrm{n}^{\mathrm{o}} 15 / 09$.

\section{LITERATURA CITADA}

AOAC. 1995. Paralytic shellfish poison. Biological method. Final action. En: AOAC (ed). Official methods of analysis, 35: 21-22. Association of Official Analytical Chemists, Gaithersburg. [sec 959.08]

Aune T. 2008. Risk assessment of marine toxins. En: Botana LM (ed). Seafood and freshwater toxins, pp. 3-20. CRC Press, Taylor \& Francis, Boca Raton.

Aune T \& M Yndestad. 1993. Diarrhetic shellfish poisoning. En: Falconer IR (ed). Algal toxins in seafood and drinking water, pp. 87-104. Academic Press, London.

Aune T, R Sørby, T Yasumoto, $H$ Ramstad \& T Landsverk. 2002. Comparison of oral and intraperitoneal toxicity of yessotoxin towards mice. Toxicon 40(1): 77-82.

Balech E. 2002. Dinoflagelados tecados tóxicos del Cono Sur Americano. En: Sar EA, ME Ferrario \& B Reguera (eds). Floraciones algales nocivas en el Cono Sur Americano, pp. 123-144. Instituto Español de Oceanografía, Madrid.

Faust MA \& RA Gulledge. 2002. Identifying harmful marine dinoflagellates. Contributions from the United States National Herbarium 42: 1-144.

Fernández ML, B Reguera, I Ramilo \& A Martínez. 2001. Toxinología y contenido tóxico de Dinophysis acuminata, D. acuta y D. caudata de las Rías Bajas Gallegas. VI Reunión Ibérica sobre Fitoplancton Tóxico y Biotoxinas, Junta de Andalucía, Congresos y Jornadas 55/00: 127-137.

Fernández ML, A Míguez, E Cacho, A Martínez, J Diogène \& T Yasumoto. 2002. Bioensayos con mamíferos y ensayos bioquímicos y celulares para la detección de ficotoxinas. En: Sar EA, ME Ferrario \& B Reguera (eds). Floraciones algales nocivas en el Cono Sur Americano, pp. 77-120. Instituto Español de Oceanografía, Madrid.

Gayoso AM \& N Ciocco. 2001. Observations on Prorocentrum lima of North-Patagonian coastal waters (Argentina) associated with a diarrhoeic disease episode. Harmful Algal News, Francia 22: 4.

Gayoso AM, S Dover, SL Morton, M Busman, PDR Moeller \& L Maranda. 2002. Possibility of diarrheic shellfish poisoning associated with Prorocentrum lima (Dinophyceae) in Patagonian Gulfs (Argentina). Journal of Shellfish Research 21: 461-463.

Hallegraeff GM \& IAN Lucas. 1988. The marine dinoflagellate genus Dinophysis (Dinophyceae): photosynthetic, neritic and not-photosynthetic, oceanic species. Phycologia 27: 25-42.

Krock B, U Tillman, U John \& AD Cembella. 2009. Characterization of azaspiracids in plankton size fractions and isolation of an azaspiracid-producing dinoflagellate from the North Sea. Harmful Algae 8: 254-263.

Lee JS, T Igarashi, S Fraga, E Dahl, $P$ Hovgaard \& $T$ Yasumoto. 1989. Determination of diarrhetic shellfish toxins in various dinoflagellate species. Journal of Applied Phycology 1(2): 147-152.

Marasigan AN, S Sato, Y Fukuyo \& M Kodama. 2001. Accumulation of a high level of diarrhetic shellfish toxins in the green mussel Perna viridis during a bloom of Dinophysis caudata and Dinophysis miles in Saipan Bay, Panay Island, the Philippines. Fisheries Science 67(5): 994996.

Masselin P, P Lassus \& M Bardouil. 1992. High performance liquid chromatography analysis of diarrhetic toxins in Dinophysis spp. from the French coast. Journal of Applied Phycology 4(4): 385-389.

Méndez S \& G Ferrari. 2002. Floraciones algales nocivas en Uruguay: antecedentes, proyectos en curso y revisión de resultados. En: Sar EA, ME Ferrario \& B Reguera (eds). Floraciones algales nocivas en el Cono Sur Americano, pp. 271-288. Instituto Español de Oceanografía, Madrid.

Park MG, S Kim, HS Kim, G Myung, YG Kang \& W Yih. 2006. First successful culture of the marine dinoflagellate Dinophysis acuminata. Aquatic Microbial Ecology 45(2): 101-106.

Raho N, G Pizarro, L Escalera, B Reguera \& I Marín. 2008. Morphology, toxin composition and molecular analysis of Dinophysis ovum Schütt, a dinoflagellate of the 'Dinophysis acuminata complex'. Harmful Algae 7(6): 839-848.

Reguera B. 2002. Establecimiento de un programa de seguimiento de microalgas tóxicas. En: Sar EA, ME Ferrario \& B Reguera (eds). Floraciones algales nocivas en el Cono Sur Americano, pp. 19-54. Instituto Español de Oceanografía, Madrid. 
Satake M, K Ofuji, H Naoki, KJ James, A Furey, T McMahon, J Silke \& T Yasumoto. 1998. Azaspiracid, a new marine toxin having unique spiro ring assemblies, isolated from Irish mussels, Mytilus edulis. Journal of the American Chemical Society 120: 9967-9968.

Scoging AC. 1998. Marine biotoxins. Journal of Applied Microbiology Symposium Supplement 84: 41S-50S.

Tillmann U, M Elbrächter, B Krock, U John \& AD Cembella. 2009. Azadinium spinosum gen. et sp. nov. (Dinophyceae) identified as a primary producer of azaspiracid toxins. European Journal of Phycology 44(1): 63-79.
Tubaro A, S Sosa, A Bornancin \& J Hungerford. 2008. Pharmacology and toxicology of diarrheic shellfish toxins. En: Botana LM (ed). Seafood and freshwater toxins, pp. 229-253. CRC Press, Taylor \& Francis, Boca Raton.

Yasumoto T, M Murata, Y Oshima, K Matsumoto \& J Clardy. 1984. Diarrhetic shellfish poisoning. En: Ragelis EP (ed). Seafood toxins, ACS Symposium Series 262: 207214. American Chemical Society, Washington.

Yasumoto T, Y Oshima, W Sugawara, Y Fukuyo, H Oguri, T Igarashi \& N Fujita. 1980. Identification of Dinophysis fortii as the causative organism of diarrhetic shellfish poisoning. Bulletin of the Japanese Society of Scientific Fisheries 46(11): 1405-1411.

Recibido el 17 de marzo de 2010 y aceptado el 2 de septiembre de 2010 